\title{
Users' experiences of enhancing underwater images: an empirical study
}

\author{
Simon Emberton ${ }^{1}\left[\right.$ Christopher Simons $^{1}$
}

Received: 27 October 2020 / Published online: 22 December 2021

(c) The Author(s) 2021

\begin{abstract}
Within the worldwide diving community, underwater photography is becoming increasingly popular. However, the marine environment presents certain challenges for image capture, with resulting imagery often suffering from colour distortions, low contrast and blurring. As a result, image enhancement software is used not only to enhance the imagery aesthetically, but also to address these degradations. Although feature-rich image enhancement software products are available, little is known about the user experience of underwater photographers when interacting with such tools. To address this gap, we conducted an online questionnaire to better understand what software tools are being used, and face-to-face interviews to investigate the characteristics of the image enhancement user experience for underwater photographers. We analysed the interview transcripts using the pragmatic and hedonic categories from the frameworks of Hassenzahl (Funology, Kluwer Academic Publishers, Dordrecht, pp 31-42, 2003; Funology 2, Springer, pp 301-313, 2018) for positive and negative user experience. Our results reveal a moderately negative experience overall for both pragmatic and hedonic categories. We draw some insights from the findings and make recommendations for improving the user experience for underwater photographers using image enhancement tools.
\end{abstract}

Keywords Image enhancement · Underwater imagery · User experience (UX)

\section{Introduction}

With the wide availability of affordable technologies for underwater photography, capturing underwater images is becoming increasing popular within the worldwide diving community. However, due to the nature of the marine environment, underwater images often suffer from degradation such as colour distortions, low contrast, blurring and occlusions caused by particles suspended in the water. Raw digital underwater images can be enhanced by various software packages (e.g. [3, 4]) to compensate for these phenomena, and also to improve the aesthetics of the image. Although feature-rich software packages and tools exist to assist underwater photographers and are widely used, the majority

Simon Emberton

simon.emberton@uwe.ac.uk

Christopher Simons

chris.simons@uwe.ac.uk

1 Department of Computer Science and Creative Technologies, University of the West of England, Bristol BS16 1QY, UK of such software applications are tailored towards enhancing terrestrial images. Furthermore, it is highly desirable that interacting with image enhancement software is experienced as useful and pleasurable by individual underwater photographers. This study aims to learn about the image enhancement tools used by the community of underwater photographers by conducting an online questionnaire. Then building on these findings, this study also aims to investigate the characteristics of the community's user experience during interaction with such tools via face-to-face interviews.

Research studies into user experience for a range of interactive software systems are widely available. However, to the best of our knowledge, there is a lack of systematic empirical investigation into (1) the tools and packages being used by the underwater photographer community, and (2) user experience during interaction with these tools for underwater photographers. To address this gap in the literature, we pose the following research questions:

RQ1 What image enhancement software tools and packages are used by underwater photographers? 
RQ2 What are the characteristics of the underwater image enhancement user experience for underwater photographers?

We note that while RQ1 is specific and directed, RQ2 is more exploratory. This is to enable an open-ended investigation of the pragmatic and emotional aspects of user experience as they relate to underwater image enhancement and also other attributes that may emerge. The exploratory nature of RQ2 also enables analysis of user perceptions and thoughts regarding good or bad user experience.

To the best of our knowledge this is the first time a study of this kind has been conducted. Contributions of the work include providing an overview of the image enhancement tools being used by the underwater photography community via an online questionnaire, and a fine-grained exploration into the user experience with these tools via face-to-face interviews (section "Methodology"). Our findings (sections "Results: online questionnaire" and "Results: faceto-face interviews") indicate a mixed picture, showing that underwater photographers are using tools designed predominantly for terrestrial imagery. There are also large discrepancies in how the tools are experienced by individual users, in particular with respect to different levels of user experience with the tools.

\section{Background}

In this section we describe software tools and packages for image enhancement, and examine the research literature with regard to user experience.

\section{Image enhancement software tools}

Underwater images suffer from specific degradations due to the way that light travels in the medium. For example, sand and other particles suspended in the water can reduce underwater visibility, similar to thick fog on land. In such conditions, underwater images can appear low in contrast and blurred due to the scattering properties of the medium [61]. Moreover, water attenuates different wavelengths of light to different degrees. Longer wavelength colours (e.g. red) are highly attenuated and fail to penetrate deep water, whereas shorter wavelengths (e.g. green and blue) are more readily transmitted [60]. The absorptive properties of the water thus cause image colour distortions. To compensate for these phenomena, underwater photographers use artificial lighting. However, this introduces non-uniform light within the scene and can further highlight particles present in the water column, normally referred to as "backscatter" [53].

Image enhancement software tools are used to remove or lessen the impact of these unwanted effects and improve the image from the aesthetic viewpoint of the underwater photographer. For example, white-balancing can be adjusted to minimise colour distortions, and dehazing and/or contrast enhancement filters can improve the image contrast. The automated enhancement of underwater images is an active research area which mainly focuses on the tasks of visibility enhancement and colour correction. In a recent survey paper [59], automated methods are categorised as:

- Model-free (e.g. histogram equalisation [66], image fusion [1]),

- Prior-based i.e. based on underwater imaging models where parameters are estimated making certain assumptions (e.g. [38, 40]),

- Data-driven i.e. neural network-based methods that learn parameters through training on large datasets (e.g. [51, 74]).

However, automated methods are currently hindered by a number of limitations. For example, individual algorithms are unable to generalise to all the possible underwater conditions, meaning that methods may perform well in some situations but poorly in others. In addition, commonly used underwater-specific image quality metrics $[64,76]$ measure the number of edges, contrast levels and colourfulness. However, artifacts and colour distortions introduced by enhancement methods can be treated as positive by the metrics even though they reduce aesthetic image quality and therefore current metrics do not always align with human subjective judgement [59].

Underwater photographers generally use image enhancement software tools and packages, including Adobe Creative Cloud, which incorporates Adobe Lightroom [3], Adobe Photoshop [4] and other application tools. Such software tools and packages are typically feature-rich and non-trivial to use, and image enhancement usually involves manually selecting parameter values which can be time consuming and repetitive. Underwater photographers have traditionally referred to image enhancement as "post-processing", in the sense of an extension of the darkroom for the film-based development process. In order to perform this task well, individuals require both a technical knowledge of the possibilities of the software as well as a subjective sensibility to achieve a desirable output. Adobe provides a number of introductory tutorials for Adobe Lightroom [30] and Adobe Photoshop [31] with a focus on land-based photography from beginner to more advanced. For underwater photography specifically, much anecdotal and grey literature is available offering advice in the form of blog posts, videos, books and white papers promoted by a variety of ventures. Examples include 5 Easy Steps to Process Underwater Photos [44], Underwater Photo Editing for Beginners [41], and Image Enhancement [42, pp. 164-180]. 
In this work we aim to get a better understanding of the range of tools being used by underwater photographers to enhance their imagery. This will be achieved through an online questionnaire targeted towards this community.

\section{User experience}

Knowledge of the user experience is essential for wide adoption of interactive software systems in user populations [50]. Providing a comprehensive user experience requires interactive software systems to be productive in terms of practical task achievement, and also address other user concerns, for example, the need to be pleasing, engaging and enjoyable. Studies taking this view of user experience have been conducted in variety of interactive system domains e.g. recommender systems [55], mobile phones [65], serious games [62], website design [68], and online banking [63].

In line with the International Standards Organisation [52], we take a perspective of user experience as a person's perceptions and responses resulting from use and/or anticipated use of a product, system, or service. We also view user experience to be subjective, context-dependent and dynamic as suggested by Law et al. [56]. The subjective nature of user experience reflects the personal diversity of users in terms of their thoughts, reflections and emotions (e.g. [70]). The context-dependent character of user experience implies that the time, place and purpose of system interaction are important (e.g. [75]). The dynamic nature of user experience suggests that an interactive experience may change over time, perhaps as users become familiar with the system, encounter difficulties or become bored or frustrated (e.g. [54]). This last point emphasises the need for interactive systems to provide users with episodes of pleasing or engaging use. For example, in the design of an ATM terminal, Tractinsky et al. [73] assert that "what is beautiful is usable", suggesting that pleasure derived from the perceived beauty of an interactive system might help to prevent user boredom or frustration in the achievement of practical tasks.

The work of Hassenzahl on the experiential element of user interaction [46-49] has attracted much research attention. Central to Hassenzahl's contribution is a distinction between pragmatic and hedonic attributes of user experience. On the one hand, Hassenzahl suggests that pragmatic attributes relate to the manipulation of software to fulfil behavioural goals. According to Hassenzahl [49, p.304], "typical pragmatic attributes of software products are clear, useful, and controllable". On the other hand, hedonic attributes are concerned with individuals' psychological well-being and pleasure. Typical hedonic attributes of software tools might include "outstanding", "impressive", "exciting" and "interesting" [49, p. 305].

Hassenzahl goes further to suggest that hedonic attributes can relate to providing stimulation, communicating identity, and evoking valued memories. For example, a user may be stimulated by novel, innovative or exciting software functionality that might also assist goal fulfilment. In addition, a software tool might communicate identity by allowing individuals to be viewed in significant ways by respected others, or might provoke pleasing memories of important past situations, relationships or thoughts. Other authors (e.g. Sheldon et al. [72]) echo the need for a pleasurable interactive experience in terms of user satisfaction, and relate this to a sense of user autonomy, competence, relatedness to significant others, and self-esteem. Hassenzahl also goes on to suggest that the balance between pragmatic and hedonic attributes of user experience can be reflected in the software product. Where hedonic attributes predominate, pleasurable aspects of the individual experience are key; where pragmatic attributes are strong, the emphasis is on behavioural goal fulfilment. However, a combination of strong pragmatic and strong hedonic attributes is desirable for a positive user experience overall.

In this study, we draw upon the pragmatic/hedonic frameworks of Hassenzahl to structure our analysis as it appears well-suited to investigating the user experiences of underwater photographers during interaction with image enhancement software tools and packages.

\section{Methodology}

In this section we describe the methodological approaches taken to address the research questions by both the online questionnaire and face-to-face interviews.

\section{Online questionnaire}

To address RQ1, the goal of our questionnaire was to gather demographic information and examples of the tools used for enhancing underwater imagery. We chose to conduct our questionnaire of underwater photographers online. The selection of appropriate participants and choice of survey questions requires careful consideration. Thus, drawing upon best survey practice (e.g. [39, 43, 45, 57]), both of these components are described as follows.

In selecting the target population of underwater photographers, we concluded that a range of practitioners from advanced beginner to expert/professional would likely have experience with image enhancement tools for underwater photography. The sampling frame of the target population was selected from members of various underwater imagery groups. To announce our research and promote interest, we attended meetings of the Bristol Underwater Photography Group, UK [37]. We also participated in virtual online communities including the British Society of Underwater Photographers [8], Scottish Underwater Photography Group 
[22], South Florida Underwater Photography Society [25], Western Australia Underwater Photographic Society [28] and Wetpixel [29]. All underwater imagery groups in the sample frame conducted their activities via the English language.

When designing our questions we wished to find out about the participants in relation to their image enhancement tool use and expertise. We posed questions relating to demographic information (i.e. age, geographic location), the participants' perception of their expertise and what various software tools they used for image enhancement. Where appropriate, we used a Likert Scale [58] with five points (e.g. 'strongly disagree', 'disagree', 'neutral', 'agree' and 'strongly agree') to enable quantitative analysis. We developed an implementation of the questionnaire using the Qualtrics platform [33].

Pretesting (see [39], Chap. 10) was conducted. Five experienced underwater photographers from the Bristol Underwater Photography Group [37] undertook a prototype questionnaire and their experiences evaluated with respect to:

1. Assimilation: could respondents satisfactorily attend to relevant questions and instructions?

2. Comprehension: could respondents understand the questions?

3. Reporting: were questions clear and did they offer an adequate opportunity for responses?

4. Appropriateness: did the questions seek appropriate and relevant information?

After pretesting was complete, we published an online link of the questionnaire to the underwater imagery groups and virtual communities we had previously contacted. The questionnaire was made available from 1st July to 31st August 2018, and attracted 81 consistent responses. We are grateful to the underwater photographers who responded. We obtained informed consent from participants prior to conducting the questionnaire and participant withdrawal was possible at any point up until data was incorporated into analysis. Consistency checks were performed to confirm that none of the responses were:

- repeats, i.e. where one respondent had not made multiple submissions,

- incomplete, i.e. where questions were not answered, and

- invalid, i.e. where responses were not reasonable replies to the questions.

Inconsistent responses were removed from the participant sample. All personal data obtained, e.g. email addresses, was entirely at the discretion of the participant. Questionnaire information was strictly confidential and stored on secure devices. We anonymised all questionnaire data prior to publication. Results and analysis of the responses are described in section "Results: online questionnaire".

\section{Face-to-face interviews}

To address RQ2, we investigated user experience during interaction with image enhancement software tools for underwater imagery by means of face-to-face interviews. We chose face-to-face interviews (rather than online) as we hoped this would create a more relaxed setting for the participants to open up about their experiences. We aimed to interview ten underwater photographers, and we selected our sample frame by choosing questionnaire respondents who used Adobe products and displayed a variety of experience with image enhancement tools. We chose questionnaire respondents who used Adobe products because as we found from the results of the questionnaire, there was a wide distribution of Adobe products among the online questionnaire participants. Due to logistical practicalities, all face-to-face interviewees were UK-based. Interviewees received a small honorarium for attending interviews (i.e. Amazon vouchers worth 20 GB pounds). They were also reimbursed for their travel expenses.

We obtained informed consent from participants prior to conducting interviews. Interviews began in a structured manner with closed-form questions (relating to participant age, gender, experience and enjoyment of enhancement software). This was followed by a semi-structured interview segment consisting of open-form questions. The open-form questions were non-leading, influencing the response as little as possible. These open-from questions thus encouraged participants to describe their user experience with image enhancement tools from their own viewpoint, and afforded opportunities for more probing follow-up questions from researchers. For example, follow-up questions might ask the participant to amplify or expand their answer, or clarify and explain their answer in more depth. Follow-up questions helped to elicit a better understanding of underlying participant values, views and experiences of underwater image enhancement. The interviews were conducted between July and December 2019.

Initially, interview participants were requested to record their age and gender. Next, participants were asked the following five closed-form questions:

1. What workstation (e.g. hardware, operating system) do you usually use?

2. What post-processing ${ }^{1}$ software tools do you usually use?

\footnotetext{
${ }^{1}$ As underwater photographers are more familiar with the term 'post-processing' than 'image enhancement', this was the terminology used in all the questions.
} 
Table 1 Categories and codes used for thematic analysis taken from Hassenzahl's first [46] and second [49] models

\begin{tabular}{lllll}
\hline Category & Code & Description & In 1st & In 2nd \\
\hline Pragmatic & Usability & Ways to access functionality & $\checkmark$ & $\checkmark$ \\
Pragmatic & Utility & Relevant functional capabilities & $\checkmark$ & $\checkmark$ \\
Hedonic & Autonomy & Independence e.g. in workflow & & $\checkmark$ \\
Hedonic & Competence & Effectiveness e.g. achieving tasks & & $\checkmark$ \\
Hedonic & Evocation & Precious memories via tool use & $\checkmark$ & \\
Hedonic & Identification & Through tool professionalism & $\checkmark$ & $\checkmark$ \\
Hedonic & Meaning & E.g. self-actualisation in tool use & & $\checkmark$ \\
Hedonic & Popularity & Influence and impact on others & & $\checkmark$ \\
Hedonic & Relatedness & E.g. contact with people & & $\checkmark$ \\
Hedonic & Security & Salient when the user senses threat & & $\checkmark$ \\
Hedonic & Stimulation & E.g. through a tool's novelty & $\checkmark$ & \\
Other & Miscellaneous & E.g. other responses of note & & \\
\hline
\end{tabular}

3. Where ' 1 ' is 'novice/amateur' and ' 5 ' is 'expert/professional', how would you rate your level of experience in underwater photography?

4. Where ' 1 ' is 'novice/amateur' and ' 5 ' is 'expert/professional', how would you rate your level of experience in using post-processing tools?

5. On a scale of ' 1 ' to ' 5 ' where ' 1 ' is no enjoyment and ' 5 ' is the most enjoyment possible, how much do you enjoy post-processing overall?

Quantitative data analysis was applied to these closed-form questions. Independent variables (i.e. age, experience with underwater photography, experience with image enhancement tools, enjoyment with image enhancement tools) were compared for any statistically significant relationships.

Interview participants were then asked the following four open-form questions related to their interaction with image enhancement tools:

6. Can you tell us what you enjoy about post-processing?

7. Are there parts of post-processing that you find particularly interesting?

8. Are there parts of post-processing that you find boring?

9. Are there parts of post-processing that you think could be improved in some way?

The ensuing interview conversations were audio recorded, transcribed verbatim, and imported into NVivo 12 software [15]. We created a pre-defined set of codes using Hassenzahl's pragmatic/hedonic frameworks $[46,49]$. Table 1 provides an overview of the coding categories that were used in this analysis. For pragmatic attributes, the Hassenzahl frameworks offer a single code of 'manipulation' of relevant product functionality. However, to analyse in greater depth, we chose to investigate the distinction between the pragmatic notions of utility (i.e. functionality) and usability (i.e. ways to access this functionality) [49]. For the hedonic attributes we made use of those mentioned in Hassenzahl's first model [46] (i.e. stimulation, identification, and evocation) as well as further attributes included in Hassenzahl's second model [49] (i.e. autonomy, competence, relatedness, popularity, security, and meaning). The attributes from the second model were developed from research into different types of motives that lead to certain types of actions [72]. We then qualitatively coded the transcript data in the form of thematic analysis [67, 69, 71].

Both of the authors individually examined all the transcripts, and identified and coded relevant textual indicators of positive and negative experience in the text which were related to either pragmatic, hedonic or other aspects. Some parts of the text were attached to multiple codes, and not all of the text was coded. The authors conducted three rounds of coding, with extensive discussions on the definitions of the codings to ensure that the codes were being appropriately assigned. As part of evaluating the codings on each round, the use of sub-codes was discussed if further detailed analysis was deemed to yield deeper insight. Results and analysis of the transcripts are described in section "Results: Face-to-face interviews".

\section{Results: online questionnaire}

A total of 81 respondents completed the questionnaire $(N=81)$. Anonymous data from questionnaire responses are available (http://researchdata.uwe.ac.uk/644/). To gain an understanding of our respondents, we first invited participants 
Table 2 Age range of questionnaire respondents $(N=81)$

\begin{tabular}{lllll}
\hline & $18-29$ years & $30-49$ years & $50-64$ years & 65 years+ \\
\hline $\begin{array}{l}\text { No. of partici- } \\
\text { pants }\end{array}$ & 3 & 34 & 33 & 11 \\
$\begin{array}{l}\% \text { of respond- } \\
\text { ents }\end{array}$ & 3.7 & 42.0 & 40.7 & 13.6 \\
\hline
\end{tabular}

Table 3 Countries of residence of respondents $(N=81)$

\begin{tabular}{ll}
\hline Country of residence & $\begin{array}{l}\text { Number of respond- } \\
\text { ents }\end{array}$ \\
\hline United Kingdom & 39 \\
United States of America & 11 \\
Ireland & 8 \\
France & 4 \\
Australia, Italy, Thailand & 3 (each) \\
Germany & 2 \\
Austria, Canada, India, Israel, Norway & 1 (each) \\
Portugal, South Africa, United Arab Emirates & \\
\hline
\end{tabular}

to state their age and location of residence. Table 2 shows the age range of questionnaire respondents. The responses show that the participants represented a wide range of ages, while most were over 29 years of age.

The geographical locations of the respondents' residence revealed 16 countries. 39 respondents reside in the UK; this may be due to the fact that the authors are UK-based and gained access to networks within local underwater photography groups. Eleven respondents reside in the USA, eight in Ireland, while other respondents reside in a further 13 countries. Table 3 shows the countries of residence of questionnaire respondents.

To address RQ1, we questioned participants on the software applications they used for enhancing underwater images. The participants were able to indicate more than one software and were not required to rank them in order of preference. All of the 81 respondents used some type of software for underwater image enhancement. There was a strong preference for Adobe products with 71 of the respondents (87.7\%) using either Adobe Lightroom or Adobe Photoshop. 57 (70.3\%) reported using Adobe Lightroom, 48 (59.2\%) Adobe Photoshop and 34 (42.0\%) both. Applications that were used by four participants each were Adobe Photoshop Elements [32] and the Nik collection [12]. Apple Aperture and GIMP [27] were used by two participants each. There were also a wide range of applications that were only used
Table 4 Operating systems used by questionnaire respondents $(N=$ 81)

\begin{tabular}{llllll}
\hline & macOS & Windows & Linux & $\begin{array}{l}\text { macOS/ } \\
\text { Win- } \\
\text { dows }\end{array}$ & $\begin{array}{l}\text { Win- } \\
\text { dows/ } \\
\text { Linux }\end{array}$ \\
\hline $\begin{array}{l}\text { No. of participants } \\
\text { \% of respondents }\end{array}$ & 49 & 35 & 4 & 2 & 1 \\
\hline
\end{tabular}

by single participants which highlights the variety of available applications. These included Affinity Photo [5], Aurora HDR [7], Darktable [9], DigiKam [10], Nikon Capture NX-D [13], Noiseless [14], PhotoLemur [17], Photomatix [16], Photovista, Picasa [18], Pixeluvo [19], PlayMemories [20], RawTherapee [21], SmartPhotoEditor [23], Snapseed [24], Tonality [26] and Microsoft Windows Photo Viewer [36].

The process of image enhancement can be aided if the captured imagery is well organised and specific images can be searched for and found when needed. Therefore, we sought to discover how participants catalogued their imagery. Most respondents also make use of Adobe products for this task, with 59 participants using Adobe Lightroom [3], 11 Adobe Bridge [2], five using Adobe Photoshop [4] and two using Adobe Photoshop Elements [32]. Three participants reported using Microsoft Photos [11] and three Apple Aperture or Photos [6]. Six individuals indicated that they did not use any specific software applications to catalogue their images.

We also sought to gain a greater understanding of the operating systems underwater imaging practitioners used for cataloguing and enhancing their images. Table 4 shows the operating systems used by the 81 questionnaire respondents. There was an almost even split between macOS and Windows with Linux being used by only a few.

In summary, users predominantly use either macOS or Windows operating systems. While a wide range of software tools are used, the findings confirm that Adobe products are widely preferred. To explore the detailed characteristics of user experience during interaction with image enhancement tools, we invited a selection of questionnaire participants with a variety of experience and who used Adobe products to attend further face-to-face interviews. We judged such participants to be representative of the wider underwater photography community with respect to tool usage.

\section{Results: face-to-face interviews}

To address RQ2, we invited ten underwater photographers who used Adobe products and of varying image enhancement experience to attend face-to-face interviews. Seven accepted our invitation. Participant responses were recorded 
Table 5 Face-to-face interview participant information responses

\begin{tabular}{llllllll}
\hline P & Age & M/F & OS & Computer & Input devices & Screen & Software \\
\hline 1 & 55 & M & macOS & M.Pro & Key/Mouse & Apple 27” & AP \\
2 & 61 & M & macOS & iMac & Key/Mouse/Pad & iMac 27” & AL/AP \\
3 & 61 & M & Windows & Dell XPS & Key/Mouse & Samsung 28” & AL \\
4 & 71 & M & macOS & M.Pro & Key/Pad & M.Pro 13” & AP/SE \\
5 & 68 & M & macOS & M.Pro & Key/Pad/Wacom & 2x Eizo 29” & AL/AP \\
6 & 69 & M & macOS & M.Pro & Key/Mouse & M.Pro 13” & AL/AP \\
7 & 64 & F & macOS & M.Pro & Key/Mouse & M.Pro 13” & AL \\
\hline
\end{tabular}

OS Operating System, M.Pro MacBook Pro, Key Keyboard, Pad Trackpad, AL Adobe Lightroom, AP Adobe Photoshop, SE Silver Efex Pro [34]

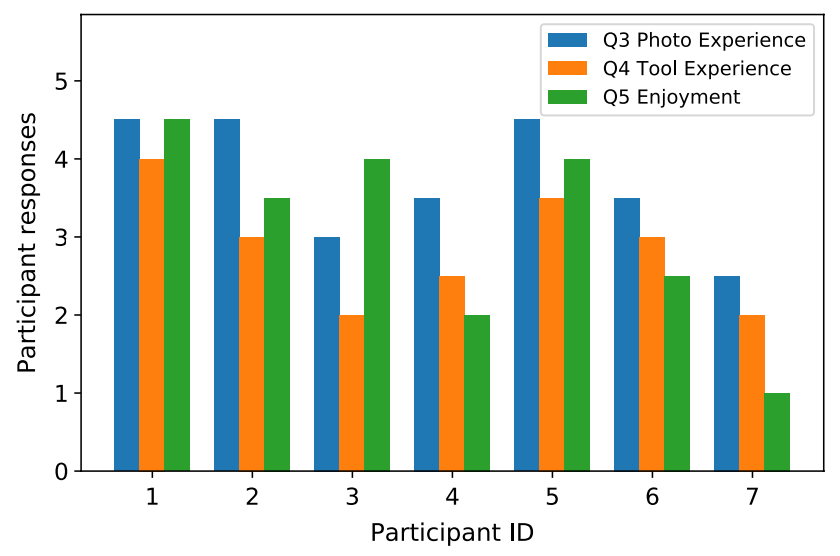

Fig. 1 Face-to-face interview participant responses to Question 3 (rating of underwater photography experience), Question 4 (rating of image enhancement tool experience) and Question 5 (rating of enjoyment when using image enhancement tools). The ratings are on a scale of ' 1 ' to ' 5 ' where ' 1 ' is the least and ' 5 ' the most experience/ enjoyment

and transcribed; interview transcripts and analysis of qualitative data are available in NVivo 12 [15] format (http:// researchdata.uwe.ac.uk/644/).

\section{About the participant sample}

Of the ten candidates invited for face-to-face interview, seven volunteered to participate. Results of interview participant responses to closed-form questions can be seen in Table 5 and Fig. 1. Table 5 shows the responses of the seven interview participants to questions relating to age, gender, and the hardware and software tools they use. We observe that the predominant gender of the participants is male, and the most common technology configuration is a MacBook Pro with Adobe Lightroom and/or Adobe Photoshop. Most users make use of standard input devices such as keyboard, mouse and/or trackpad, although one participant (P5) did also make use of a Wacom tablet and pen [35]. The average age of participants is 63.2 years, which might indicate a high level of experience, although a range of participant tool experience ratings (see Fig. 1) from '2' to '4' can be seen. All of the interview participants are based in the UK and while this may mean that they dive more often in UK waters, many participants mentioned taking frequent trips abroad for underwater photography.

When interview participants were asked a question regarding their experience inviting a response from the Likert scale i.e. ' 1 ' to ' 5 ', it is interesting to note that some responded with " 2 to 3 ", "3 to 4" or "4 to 5". No respondent rated their experience as a ' 5 '. We speculate that the interview respondents experienced some difficulty in rating their own experience, and so preferred to express a small range instead. Where this occurred, we show responses as the mid-point of the range, e.g. where a respondent rated their experience as " 4 to 5 ", we show this as ' 4.5 '. Figure 1 shows participant responses to the Likert Scale closed-form questions 3, 4 and 5. Participant responses can range from ' 1 ' (lowest rating) to ' 5 ' (highest rating). Responses to Question 3 show a range of self-rated levels of experience with underwater photography from ' 2 ' to ' 4.5 '. There is also a range in the values for self-ratings for experience with image enhancement tools and packages from ' 2 ' to ' 4 '. However, the greatest range of responses can be found in relation to Question 5, i.e. the level of enjoyment when using image enhancement software tools. A range from ' 1 ' to ' 4.5 ' can be seen, indicating quite different levels of enjoyment among the participants.

We wished to compare samples of the interview participants and online questionnaire respondents. To explore this, we compared (i) age distributions, (ii) operating system used, and (iii) experience self-ratings for both.

We found the age distribution of the questionnaire respondents to be different to that of face-to-face interview participants. For instance, in the online questionnaire, 54.3\% of respondents are aged 50 years or older (i.e. $40.7 \%$ are aged between 50 and 64 years, and $13.6 \%$ are aged 65 years or older). In face-to-face interviews, the youngest participant is aged 55 years, and $42.8 \%$ are older than 65 . Regarding 


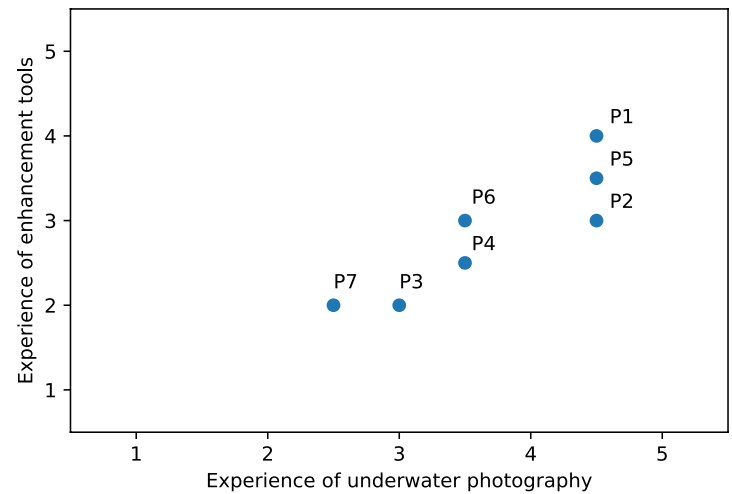

(a) Interview participants.

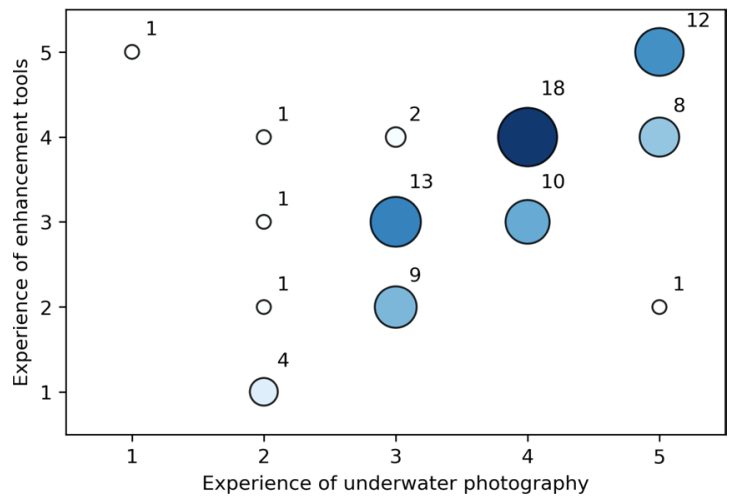

(b) Questionnaire respondents.
Fig. 2 Plots of participant ratings of underwater photography experience versus enhancement tool experience for a interview participants and $\mathbf{b}$ questionnaire respondents. Participant ratings are on a scale of

hardware used, $48.1 \%$ of online questionnaire respondents used Apple with a further $2.5 \%$ using both Apple and Windows. However, in face-to-face interviews, $85.7 \%$ of participants used Apple, with one participant using a Windows computer.

Regarding experience self-ratings of underwater photography and software enhancement tools, we plotted both for interview participants and questionnaire respondents. Figure 2a shows a plot of experience for interview participants with a Pearson's Correlation of 0.885 , statistically significant at the $p<0.01$ level, while Fig. 2b shows a plot for questionnaire respondents with a Pearson's Correlation of 0.675 , again significant at the $p<0.01$ level. Both scatter plots show a positive correlation indicating that increasing underwater photography experience is consistent with increasing enhancement tool experience.

In summary, the interview participants are on average older and more of them use Apple hardware in comparison to the questionnaire participants. Whilst the interview sample is not completely representative of the questionnaire sample both display a wide range of experience self-ratings (see Fig. 2) and are drawn from underwater imaging groups.

Comparison of participant responses for their rating of experience with image enhancement tools and their rating of enjoyment showed a degree of correlation. As Fig. 3 illustrates, responses for image enhancement tool experience correlate with rating of enjoyment (Pearson's Correlation 0.711). However, as the correlation was not statistically significant, we examined the responses of Participant three. At interview, Participant three indicated that although they had been diving underwater for many years, their experience of underwater photography and image enhancement tools was limited as they had only embarked on taking and enhancing underwater images a
' 1 ' to ' 5 ' where ' 1 ' is the least experience and ' 5 ' the most. In $\mathbf{b}$, the size of the bubble and intensity of colour reflect the number of respondent responses at the $\mathrm{x}, \mathrm{y}$ coordinate

few months prior to interview. Participant three reported they were enjoying the novelty of 'beginner' use of image enhancement tools. Excluding Participant three as an outlier, examining enhancement tool experience versus level of enjoyment reveals a positive correlation (Pearson's Correlation 0.958) which is significant at the $p<0.01$ level, albeit with six data points. These results suggest that among the interview participants, levels of enjoyment tend to increase with increasing levels of experience of image enhancement tools.

\section{Analysis of interviews}

Here we analyse the interview transcripts and describe participant reports of positive and negative user experience using the Pragmatic and Hedonic categories of the Hassenzahl frameworks. We also examine the count of codings for

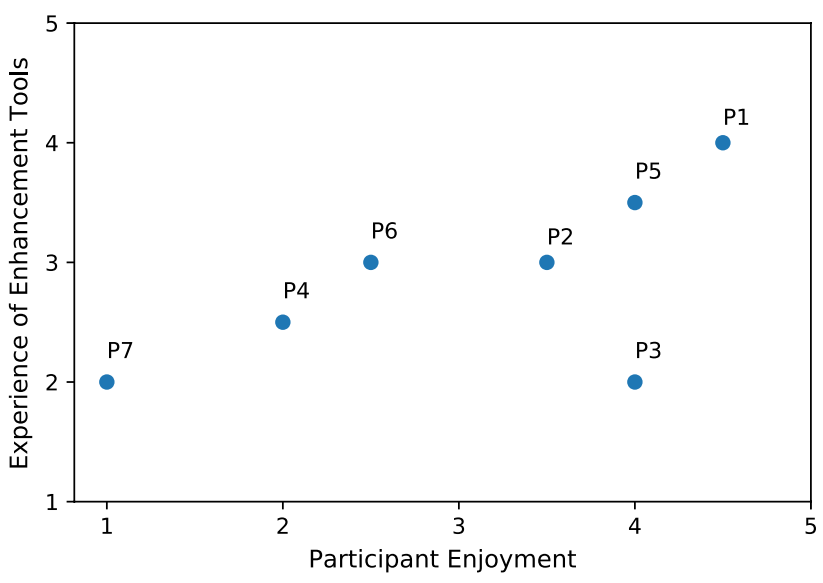

Fig. 3 Scatter plot of interview participant enjoyment rating versus experience of enhancement tools. The scale of ratings is ' 1 ' to ' 5 ' where ' 1 ' is the least and ' 5 ' the most experience/enjoyment 
Table 6 Categories and codes for positive user experience reports, with brief code descriptions and frequencies $(N$ $=83$ )

\begin{tabular}{lllll}
\hline Category & Code & Brief code description & Freq. (\%) & Freq. (\%) \\
\hline Pragmatic & & & 31.4 & \\
& Usability & Ways to access functionality & & 13.3 \\
& Utility & Relevant functional capabilities & & 18.1 \\
Hedonic & & & 67.5 & \\
& Autonomy & Independence e.g. in workflow & & 3.6 \\
& Competence & Effectiveness e.g. achieving tasks & 19.3 \\
& Evocation & Precious memories via tool use & 12.0 \\
& Identification & Through tool professionalism & & 6.0 \\
& Meaning & E.g. self-actualisation in tool use & & 2.4 \\
& Popularity & Influence and impact on others & & 3.6 \\
& Relatedness & E.g. contact with people & & 0.0 \\
& Security & Salient when a user senses threat & & 1.2 \\
Other & Stimulation & E.g. through a tool's novelty & & 19.3 \\
& & & 1.2 & 1.2 \\
\hline
\end{tabular}

each participant using the frameworks, and analyse this with respect to the participants' experience with image enhancement tools.

\section{Positive user experience}

Table 6 shows the frequencies of coded participant responses, with respect to a positive user experience. The Pragmatic category is comprised of two codes, the Hedonic category nine and the Other category is comprised of just one code (miscellaneous). The category with the most frequently occurring codings is Hedonic $(67.5 \%)$, in comparison to Pragmatic (31.4\%) and Other (1.2\%), suggesting that an emotional user response is a very important part of the positive experience.

The two most frequently occurring codes are hedonic competence (19.3\%) and hedonic stimulation (19.3\%). This suggests that in terms of an emotional response, users can feel positive about both their competence to use image enhancement software to achieve tasks, and being stimulated by the software to, for example, create novel underwater images. To give a sense of this, examples of responses coded as competence and stimulation respectively include:

"You can have an image that perhaps you've horribly underexposed. If you've shot it in raw and then you really can often salvage quite a decent image out of it, that can be enjoyable if you get something that you weren't expecting." (P4)

"I think what's really enjoyable for me is to be able to visualise, to pre-visualise, what it is I'm looking for and then to actualise my vision right in front of a computer so I created something. I made a picture, I made a photograph and then knowing the tools that were at my disposal, I was able to enhance that to a point where my vision came to life so for me it's massively enjoyable." (P1)

The third and fourth most frequently occurring codes are pragmatic utility (18.1\%) and pragmatic usability (13.3\%), respectively. This suggests that the functional aspects of image enhancement tools, as well as ways to access that functionality, are also important for a positive user experience. As an illustration of a positive pragmatic utility response, Participant five observed that:

"I can do so much more with the tools that are available now." (P5)

As an example of a positive pragmatic usability response Participant five also commented that:

"You can do these things quite quickly you know just put a grad filter on pretty quickly in Lightroom." (P5)

Other participant coded responses occur less frequently and are mostly hedonic. For example, the code evocation was recorded with a frequency of $12.0 \%$. One participant described a strongly positive sense of evocation when describing their experience of image enhancement software as a "digital darkroom" as follows:

"It's part of the process of producing a photograph, it's as much a part as getting the composition right underwater and exposure and all that kind of thing but to me you're only halfway there when you've done that. I've got the other half to do when I'm back home and I think that attitude comes out of where I come from because I spent hours and days in the 
Table 7 Categories and codes for negative user experience reports, with brief descriptions and frequencies $(N=98)$

\begin{tabular}{lllll}
\hline Category & Code & Brief description & Freq. (\%) & Freq. (\%) \\
\hline Pragmatic & & & 35.7 & \\
& Usability & Ways to access functionality & & 16.3 \\
& Utility & Relevant functional capabilities & & 19.4 \\
Hedonic & & & 64.3 & \\
& Autonomy & Independence e.g. in workflow & & 10.2 \\
& Competence & Effectiveness e.g. achieving tasks & 20.4 \\
& Evocation & Precious memories via tool use & 2.0 \\
& Identification & Through tool professionalism & & 9.2 \\
& Meaning & E.g. self-actualisation in tool use & & 0.0 \\
& Popularity & Influence and impact on others & & 2.0 \\
& Relatedness & E.g. contact with people & & 0.0 \\
& Security & Salient when the user senses threat & & 0.0 \\
Other & Stimulation & E.g. through a tool's novelty & & 20.4 \\
& & & 0 & \\
& Miscellaneous & Other negative responses of note & & 0.0 \\
\hline
\end{tabular}

darkroom as a teenager and a young adult the darkroom work and enhancing images and so on." (P5)

Remaining hedonic codes (autonomy, identification, meaning, security, relatedness) occur with a frequency of $6.0 \%$ or less, suggesting that these are not prominent characteristics of a positive user experience with the software. There were no instances of the coding category relatedness, which also suggests that a sense of contact with other people for whom the user cares does not feature as part of the positive user experience. One instance of a positive experience that could not be assigned to any of the pragmatic or hedonic codes was identified. This experience related to a sense of progress towards the end goal i.e.:

"The path along the way that each individual step takes me closer to an end result and it's the end result that I enjoy." (P1)

\section{Negative user experience}

Table 7 shows the frequencies of codings of participant responses with respect to reports of negative user experience with image enhancement software tools. The majority of negative codes are reported for the Hedonic category with a total of $64.3 \%$ in comparison to $35.7 \%$ for the Pragmatic category and none for Other. Similarly to the reports of positive user experience, the most frequently occurring negative codes are hedonic competence (20.4\%) and hedonic stimulation $(20.4 \%)$.

Users report negative hedonic experiences with the software in regards to their competence. This is illustrated in the following statement of Participant three:
"I mean it's a complicated process with a lot of language to it. I guess like anything really once you know it you know it but there is a lot to learn and it requires a lot of time." (P3)

There were also a large number of reports of negative hedonic stimulation which indicates that users may not find the use of these tools particularly interesting or enjoyable. This is characterised in the following response of Participant six:

"I have to force myself to do it, so it's not that I find it boring, it's tedious, and I usually fall asleep after three quarters of an hour." (P6)

Participant five also reported underwater backscatter removal (i.e. cloning out individual instances of backscatter) as negatively stimulating:

"Backscatter removal, boring boring boring." (P5)

The relatively high frequency of the reports of pragmatic utility (19.4\%) and pragmatic usability (16.3\%) demonstrate that negative user experience is also influenced by the functional aspects of the tools and the ways to access that functionality. This is shown in the following negative pragmatic utility response from Participant one:

"You have to be careful you have to use these tools sparingly so you don't, okay, make it look too synthetic." (P1)

An example of a negative pragmatic usability response is the following from Participant two:

"I'll have forgotten how to do it when it comes around again because it's so few and far between and I'll have 
Table 8 Pre-defined codes (usability and utility) and sub-codes developed during analysis of positive and negative pragmatic reports $(N=61)$

\begin{tabular}{|c|c|c|c|c|}
\hline Code & Sub-code & Brief description & Freq. $(\%)$ & Freq. $(\%)$ \\
\hline \multirow[t]{3}{*}{ Positive usability } & & & 18.0 & \\
\hline & Enabling & Enabling analysis and understanding & & 9.8 \\
\hline & Quick/easy (Adobe Lightroom) & Performing tasks quickly and easily & & 8.2 \\
\hline \multirow[t]{9}{*}{ Positive utility } & & & 24.6 & \\
\hline & Aesthetic improvement & Aesthetically enhancing images & & 9.8 \\
\hline & Amazing Adobe Photoshop & Amazing functionality in Adobe Photoshop & & 3.3 \\
\hline & Cataloguing (Adobe Lightroom) & Cataloguing functionality & & 1.6 \\
\hline & Dehaze & Removing haze & & 1.6 \\
\hline & Graduated filter (Adobe Lightroom) & Graduated neutral density filter effect & & 1.6 \\
\hline & Spot healing (Adobe Photoshop) & Removing small imperfections & & 1.6 \\
\hline & Star rating (Adobe Lightroom) & Users rate their images (scale $1-5$ ) & & 1.6 \\
\hline & Texture & Increasing medium size texture & & 3.3 \\
\hline \multirow[t]{6}{*}{ Negative usability } & & & 26.2 & \\
\hline & Accessibility & Inaccessible for visually impaired users & & 1.6 \\
\hline & Boring/frustrating & Tools are boring and/or frustrating & & 8.2 \\
\hline & Laborious (Adobe Photoshop) & Adobe Photoshop is laborious to use & & 3.3 \\
\hline & Learning/remembering & Tools hard to learn and remember & & 1.6 \\
\hline & Time consuming & Tasks take a long time & & 11.5 \\
\hline \multirow[t]{11}{*}{ Negative utility } & & & 31.1 & \\
\hline & Auto-enhance & Limited results with auto-enhance & & 3.3 \\
\hline & Auto-remove backscatter & Desire for automatic removal of backscatter & & 1.6 \\
\hline & Choosing images to process & Desire for help in choosing images & & 6.6 \\
\hline & Clone stamp tool (Adobe Photoshop) & Clone stamp tool in Adobe Photoshop & & 1.6 \\
\hline & Correct focus & Desire for focus correction & & 1.6 \\
\hline & Cut out object from background & Desire for better tools for cutting out objects & & 1.6 \\
\hline & Dehaze & Unnatural/synthetic results with dehaze tool & & 3.3 \\
\hline & Graduated filter & Difficult to achieve linear spread with filter & & 1.6 \\
\hline & Inconsistent results & Results can be inconsistent & & 3.3 \\
\hline & Limited functionality (Adobe Lightroom) & Adobe Lightroom is limited w.r.t. Adobe Photoshop & & 6.6 \\
\hline
\end{tabular}

Frequencies are given in percentages

to go through the same process of learning what I need to do and then doing it." (P2)

The next two most commonly occurring negative coding categories are hedonic autonomy at $10.2 \%$ and hedonic identification at $9.2 \%$. Participants report negative feelings of hedonic autonomy which indicates there are instances where the tools do not allow them to do things the way that they would want to. The negative reports of hedonic identification are related to how the tool facilitates their ability to feel like or be seen like a "serious" or "creative" underwater photographer.

There were very few negative reports of the hedonic coding categories evocation and popularity with both at $2.0 \%$. There were no reports at all for hedonic meaning, relatedness and security which suggests these are not currently important aspects in relation to the negative user experience of image enhancement tools.

In further addressing the second research question posed, i.e. "What are the characteristics of the underwater image enhancement user experience?", we also consider the characteristics of underwater image enhancement user experience firstly through a Pragmatic category focus, then secondly Hedonic.

\section{Pragmatic characteristics}

Pragmatic usability and utility codings for positive $(N=83)$ and negative $(N=98)$ participant responses can be seen in Tables 6 and 7, respectively. For pragmatic usability, the frequency for positive reports is $13.3 \%$ and for negative reports slightly higher with $16.3 \%$. For pragmatic utility, the frequency for positive reports is $18.1 \%$ 
and for negative reports very slightly higher with $19.4 \%$. Interview participants appear to be impressed with some of the capabilities of the image enhancement software.

"It's just how amazing Photoshop is at being able to do something that you wouldn't have thought possible." (P2)

However, further interview responses appear to indicate difficulties in achieving some practical tasks:

"Sometimes it works, sometimes it doesn't." (P2)

These results suggest that the characteristic of the user experience with respect to the pragmatic aspects of image enhancement software is slightly more negative than positive and with the functionality (utility) being reported slightly more often than ways to access the functionality (usability).

To take a closer look within the pre-defined codes of pragmatic usability and utility, sub-codes were developed during the analysis. Table 8 shows the sub-codes and reported frequencies in percentages $(N=61)$. These sub-codes indicate which specific aspects of the usability and utility of the tools are being reported as either positive or negative.

For the positive pragmatic reports, the most commonly occurring sub-codes are utility-Aesthetic improvement (9.8\%), usability—Enabling (9.8\%) and usability—Quick/ easy Adobe Lightroom) (8.2\%). The sub-code utilityAesthetic improvement contains positive user reports of tool use for making aesthetic improvements to underwater images. As an example of an utility-Aesthetic improvement response, Participant one reported using the utility of image enhancement tools to express patterns of good underwater image composition, such as the application of the 'rule of thirds ${ }^{2}$ underwater, or the diver's model facing the photographer and engaging in eye contact, with clear separation of the subject (in this case the diver's model) from the background. For example:

"I knew that my composition was effective. You know, I've got a foreground subject here which is sort of in the rule of thirds. I've got another subject here which is also at that intersection and the line is on the rule of thirds." (P1)

"And I had a very good model who I had worked with for years. She knew exactly where to pose, she can see her reflection in my dome port she knows exactly where to position herself. You can see she's not touching the edge, I framed this right..." (P1)

\footnotetext{
2 The 'rule of thirds' is a visual arts guideline relating to the composition of images. It suggests that an image should be conceived by dividing it into nine equal parts through placing two equally spaced horizontal lines and two equally spaced vertical lines, with image subject(s) aligned to the lines, and particularly at the intersections.
}

The sub-code usability—Enabling comprises responses that indicate the tools enable analysis, actualisation and an increased understanding of both the enhancement and capture of underwater images. A response from Participant three expresses an example of this sub-code as follows:

“...I try to use the editing process to to help me understand why the pictures didn't come out perfect from the camera so one thing I think is a lot of my pictures are too dark so what does that tell me about how I take them ..." (P3)

The sub-code usability-Quick/easy (Adobe Lightroom) contains frequent mentions that Adobe Lightroom in particular allows users to perform tasks quickly and easily. An example response of this sub-code from Participant five is as follows:

"I like Lightroom because it's quite easy and it's quite quick and I feel sorry for people who were brought up in Photoshop who can't make the migration to Lightroom because I just think that Lightroom is so much easier and quicker for me anyway ..." (P5)

Lesser occurring positive sub-codes with a frequency of $3.3 \%$ or less all relate to utility, indicating favourable responses of specific functionality for the task of underwater image enhancement. Users described Adobe Photoshop as providing amazing functionality (utility-Amazing Adobe Photoshop (3.3\%)) and that the spot healing tool was much better in Adobe Photoshop than Adobe Lightroom for the task of backscatter removal (utility - Spot healing (Adobe Photoshop) (1.6\%)). Users' positive reports for Adobe Lightroom mentioned the graduated filter (utility-Graduated filter (Adobe Lightroom) (1.6\%)) and the cataloging functionality (utility-Cataloguing (Adobe Lightroom) (1.6\%)) in particular the use of adding star ratings to underwater images (utility-Star rating (Adobe Lightroom) (1.6\%)). Other functionality that was positive for both Adobe Lightroom and Adobe Photoshop included the dehaze tool (utility-Dehaze (1.6\%)), for removing unwanted haze present in the water column between the photographer and the subject, and the texture tool (utility-Texture (3.3\%)) which is useful for underwater photographers to increase the level of texture in local regions that may have been lost due to the effects of the medium.

For the negative pragmatic reports the most frequently occurring negative sub-code is usability-Time consuming $(11.5 \%)$ indicating that the tools require users to spend longer than they would like performing tasks such as organising images and deciding which ones to process as well as wasting time processing images which end up with unsatisfactory results. The second most frequently occurring negative sub-code is usability-Boring/frustrating 
$(8.2 \%)$ which suggests users often find the tools boring and/or frustrating to use. Responses to this sub-code mentioned the underwater image enhancement task of backscatter removal to be particularly boring and frustrating. The joint third and fourth most frequently occurring negative sub-codes are utility-Choosing images to process (6.6\%) and utility-Limited functionality (Adobe Lightroom) (6.6\%). Participants require tools which assist in choosing which underwater images to process. This is particularly the case for underwater photographers as they often return from trips with thousands of images and are unsure which ones to spend the time processing. Users also reported that Adobe Lightroom (in comparison to Adobe Photoshop) is limited in some of the functionality commonly used by underwater photographers such as spot healing, cloning and content-aware fill. Lesser occurring negative sub-codes, with a frequency of $3.3 \%$ or less, were present for both usability (three sub-codes) and utility (eight sub-codes). In regards to usability, users reported that Adobe Photoshop can be laborious to use (usability-Laborious (Adobe Photoshop) (3.3\%)). It was also mentioned that both Adobe Photoshop and Adobe Lightroom can be difficult to learn and remember (usability_Learning/remembering (1.6\%)) and can be inaccessible for users with visual impairments (usability-Accessibility (1.6\%)). In regards to utility, users reported limitations using the auto-correction functionalities of current tools with underwater images, which aim to either adjust tones (i.e. contrast, brightness, highlights and shadows) or white balance (utility-Auto-enhance (3.3\%)). The dehaze tool (utility-Dehaze (3.3\%)) can introduce unnatural/synthetic results into underwater images. It can also be difficult to achieve an imperceptible linear spread with the graduated filter (utility-Graduated filter (1.6\%)). Users reported a lack of functionality for correcting out of focus shots (utility-Correct focus (1.6\%)). One response mentioned a desire for being able to automatically remove backscatter (utility-Auto-remove backscatter (1.6\%)). A participant negatively described the clone stamp tool (utility-Clone stamp tool (Adobe Photoshop) (1.6\%)) in Adobe Photoshop which is used for removing unwanted objects and another reported a desire to more easily cut out objects from the background (utility-Cut out object from background (1.6\%)). Users also stated that results can be inconsistent when working with underwater images (utility - Inconsistent results (3.3\%)).

With regard to the negative pragmatic aspects of user experience with underwater image enhancement tools, analysis of the discourse of one participant appears to show a possible interaction of utility and usability categories. Participant six observes that:

"Part of the issue is that it is a bit like gardening, it never ends. I could spend hours and hours and hours and produce something which is really good but I could only do it for a half a dozen photos by doing not a quick and dirty, but a limited time, I can get a product which pleases me and is appealing and technically competent even if it isn't going to win the competition." (P6)

Participant six indicates that they seek to enhance underwater images with respect to both technical competence (i.e. the extent to which typical image degradation due the marine environment has been corrected) and aesthetic appeal. However, with the emergence of ten negative utility codes during analysis, such utility shortcomings may in some way be contributing to negative usability, for example in terms of codes usability-Time consuming and usability_Boring/ frustrating.

\section{Hedonic characteristics}

In a comparison of the positive and negative hedonic responses (see Tables 6 and 7) the two most commonly occurring categories for both were hedonic competence and stimulation. The frequency of positive user experience for both hedonic competence and stimulation is $19.3 \%$ while the frequency of negative user experience for both hedonic competence and stimulation is $20.4 \%$. These results are in line with the findings for pragmatic codings (usability and utility) which also show slightly higher levels of negative user experience.

A more in-depth look at the positive and negative reports for hedonic competence show that participants like being able to use their knowledge about what will make an underwater image look better and then apply their skills in realising that goal:

"I find it a challenge so it can be it can be a challenge to bring something out of a not very good image and try and get something that's worth showing people." (verbatim transcription, $\mathrm{P} 2$ )

One participant reported reaching a stage where their mastery of the tools had meant aspects of tool use had become internalised:

"You start to actualise, and you start to enjoy that because it becomes unconsciously competent." (P1)

In contrast, participants reported negative competence with the tools, especially with the more advanced features of Adobe Photoshop which may only be used occasionally, and also choosing which underwater images to enhance. Another aspect that participants dislike is that there is much to learn and remember. However, this might also be seen as a pragmatic issue, i.e. is the tool difficult to use or is the user not sufficiently well practised? 


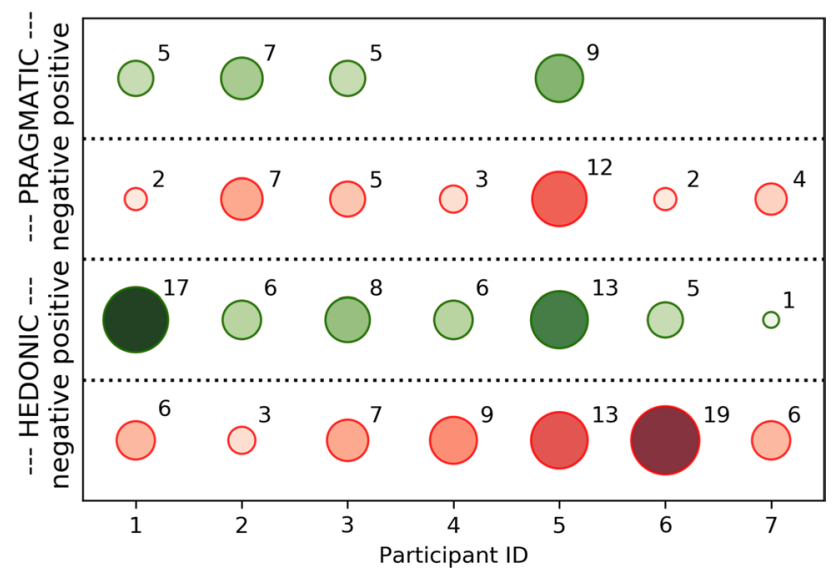

Fig. 4 Count of coding instances for each interview participant for pragmatic/hedonic, positive/negative codings. Positive codings are shown in green; negative in red. The size of the bubble and intensity of colour reflect the instance count

Positive aspects of hedonic stimulation were when the experience was challenging and allowed users to experiment, learn and understand. Users were also stimulated by the fact that the tools are constantly improving and allowing them to do new things. Negative aspects of hedonic stimulation were reported in regards to user experience when enhancing underwater imagery. Issues relate to the time it takes to do a task as well as the time it takes to learn how to do a task and having to stop what you are doing to find this out. Repetitive tasks such as marine backscatter removal are seen as "annoying", "boring" and "tedious". Another activity which provides negative stimulation is having to deal with such large volumes of images which includes sifting through thousands of underwater images, deciding which ones to process and then the time it takes to process them.

There were hedonic responses that also differed in regards to reports of positive and negative user experience. Participants reported positively in the hedonic evocation category where the frequency of positive user experience is $12.0 \%$ while the frequency of negative user experience is only $2.0 \%$. Participant's reports of positive hedonic evocation were cases where the tool use either brought back memories of when the photograph was captured and how they experienced that scene at the time:

"The tool is enhancing it. It's pulling out information that I saw. This is what it looked like to me when I was underwater. What I'm looking at there, my finished product, is what I saw underwater." (P1)

Some participants were taken back even further as the tool use reminded them of when they processed photographic film in a darkroom.

Participants reported negatively in the hedonic autonomy category where the frequency of negative user experience

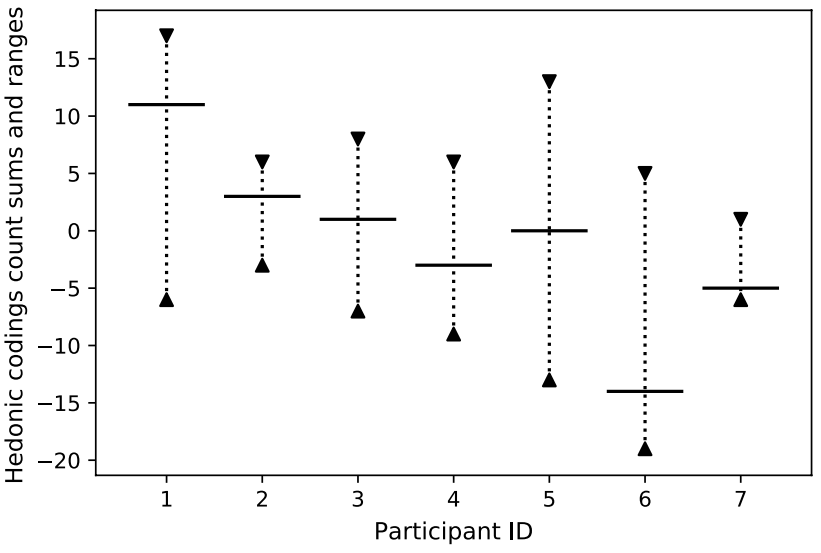

Fig. 5 Sum of count of positive hedonic coding instances minus count of negative hedonic coding instances as horizontal lines, with ranges, for each participant

is $10.2 \%$ whereas the frequency of positive user experience is only $3.6 \%$. This was mainly to do with time-consuming repetitive tasks such as marine backscatter removal and deciding which underwater images to process.

There were a few reports of both positive $(6.0 \%)$ and negative $(9.2 \%)$ hedonic identification. Positive aspects related to identifying as a famous artist (e.g. photographer or painter) and getting external recognition which confirms this identification. Negative aspects were reported for participants who didn't see themselves as being creative or as good as other underwater photographers and received negative criticism at competitions for inadequate use of the tools, thereby confirming this identification.

\section{Counts of codings in interview transcripts}

Figure 4 shows the count of coding instances for each participant across pragmatic and hedonic categories, for both positive and negative user experiences. We note that some participants' interview transcripts i.e. participants one, five and six, give rise to a higher number of coding instances than others. We speculate that participants one, five and six were possibly more articulate, and/or expanded on their answers to the open-form questions for longer periods of time. The interview responses of Participant five attracted the most coding instances with 46 , while the responses of Participant seven attracted the fewest with 11. Codings for participants one and six appear to indicate an enjoyable and unenjoyable user experience respectively.

With regard to the count of positive and negative pragmatic codings, no trends are immediately apparent in Fig. 4. However, for hedonic codings, a range in participant coding instances is evident. For each interview participant, we summed the count of negative hedonic codings with the count of positive codings. Results reflecting the sum and 


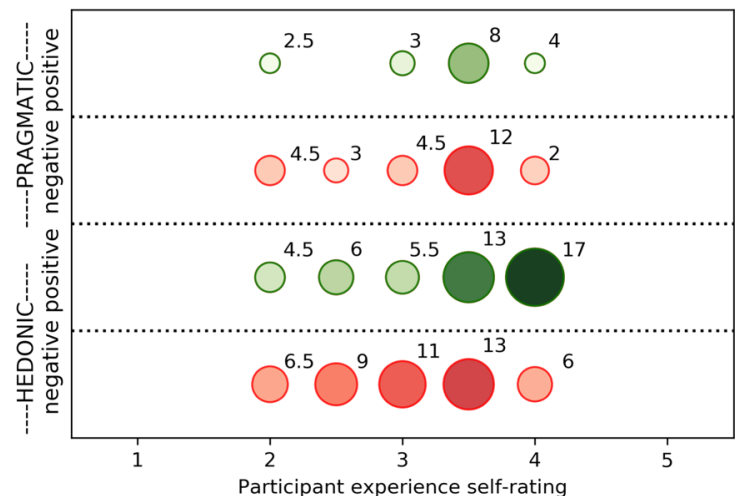

(a) All interview participants.

Fig. 6 Bubble plots showing interview participant underwater photography experience versus average coding instances. Positive codings are shown in green; negative in red. The size of the bubble and

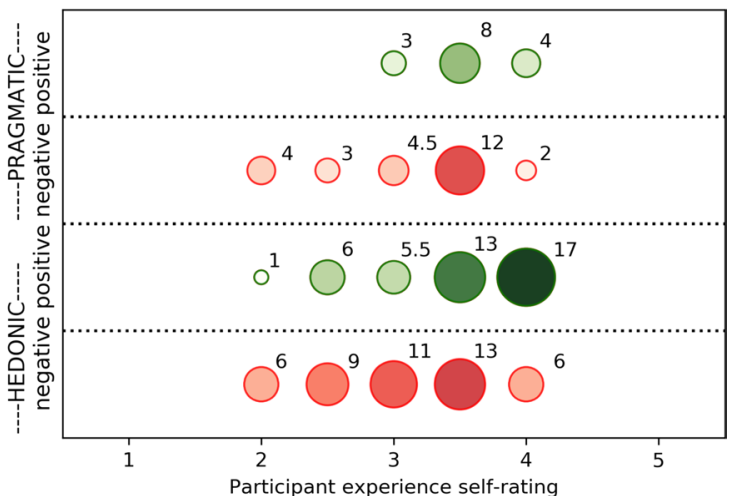

(b) Participant 3 excluded.

intensity of colour reflect the count of codings. Participant ratings are on a scale of ' 1 ' to ' 5 ' where ' 1 ' is the least experience and ' 5 ' the most. In b, outlier interview Participant three is excluded

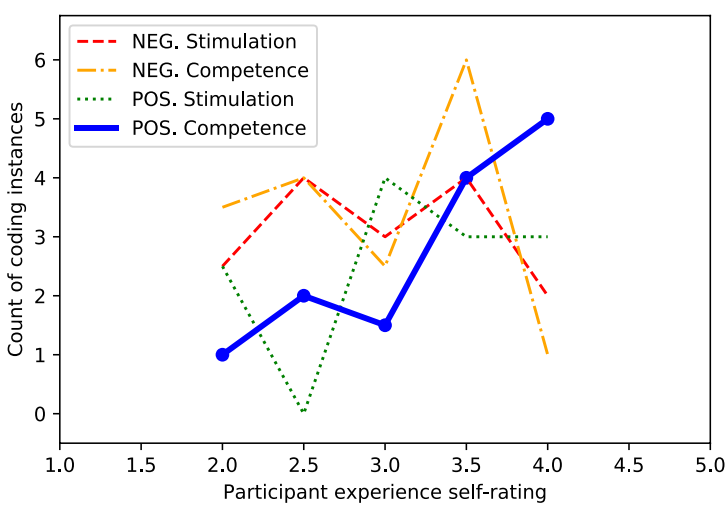

(a) All interview participants.

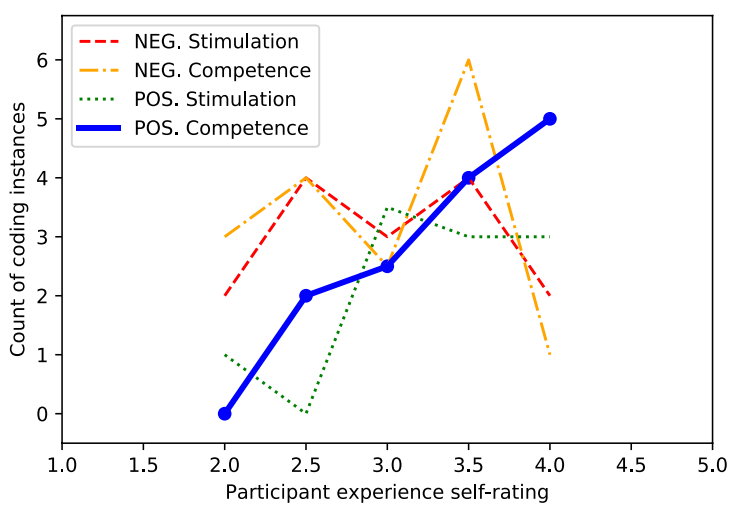

(b) Participant 3 excluded.

Fig. 7 Plots of instances for positive and negative stimulation and competence codes against participant experience. Participant ratings are on a scale of ' 1 ' to ' 5 ' where ' 1 ' is the least experience and ' 5 ' the most. In $\mathbf{b}$, outlier interview Participant three is excluded

range of hedonic coding counts are visualised in Fig. 5 and suggest a pleasant and an unpleasant user experience for participants one and six respectively. The range of codings for Participant five, on the other hand, suggests an ambivalent experience, while the range of codings for Participant two might suggest a neutral to mildly positive experience in terms of pleasure.

We also sought to examine if the count of coding instances might reflect interview participant experience with image enhancement software tools. Figure 6a shows the number of coding instances for pragmatic/hedonic categories of positive and negative user experiences based on participant experience of image enhancement tools. Some participants self-rated the same level of experience. Where this occurred, we took the average of the count of coding instances for the experience level to maintain comparability across experience levels. No trends in Fig. 6a are immediately apparent, other than a possible increase in hedonic codings with increasing experience. However, as discussed in section "About the participant sample", Participant three could be considered an outlier with respect to experience of underwater enhancement tools. Thus Fig. $6 \mathrm{~b}$ shows the number of coding instances excluding Participant three. A broad increase in the number of hedonic coding instances with participant experience then becomes apparent (albeit non-monotonic). The highest counts of coding instances for the positive hedonic category are evident for the highest experience level. This suggests that user enjoyment using image enhancement tools increases with increasing levels of experience. This is consistent with results obtained in section "About the participant sample".

We further sought to examine if the count of coding instances for the most frequent individual codes i.e. competence and stimulation might reflect participant experience with image enhancement tools. Figure 7a shows the count of coding instances for positive and negative competence and 
stimulation codes. An overall increase in instances of positive competence with participant experience is evident; no trends are immediately apparent for other codes. Excluding Participant three as an outlier, Fig. 7b shows a monotonic increase in instances of positive competence with participant experience. This suggests that as increasing experience of image enhancement tools for underwater images is acquired, a positive sense of competence is increasingly a characteristic component of the user experience.

\section{Insights and recommendations}

From the results of the analysis of the qualitative data and counts of coding instances, we draw the following insights into the characteristics of user experience with image enhancement tools for underwater images:

1. A moderately negative picture of user experience: Image enhancement tools appear to elicit a moderately negative user experience overall for underwater images, with $54.5 \%$ of all coding instances observed during analysis indicating a negative experience compared to $45.5 \%$ for a positive experience.

2. Pleasure is significant to user experience: While pleasure and functionality both contribute to the overall user experience, $66.1 \%$ of all coding instances observed during analysis relate to hedonic codings, whereas approximately half of that number (i.e. $33.8 \%$ ) relate to pragmatic codings.

3. Pragmatic utility challenges for underwater images: A number of negative pragmatic codes were discovered during analysis relating to participant attempts to address image degradation due to colour distortions, low contrast, blurring and occlusions caused by particles present in the marine environment. For example, analysis of participant transcripts revealed a number of negative pragmatic utility codes such as utility-Dehaze, utility—Focus correction, and utility—Auto-backscatter removal.

4. Stimulation and competence are crucial: Within the hedonic category, stimulation and competence emerge as the most frequent codes. Moreover, a mixed picture is evident for both codes with frequencies of codings broadly similar for positive and negative experience. The wide range of tool capabilities can provide positive stimulation for advanced users to learn new features and challenge themselves. However, for those users with less experience this large array of possibilities in the tools creates feelings of negative competence.

5. Enjoyment correlates with experience: Levels of enjoyment in the user experience characteristically appear to positively correlate with increasing levels of individuals' experience of image enhancement tool use.
This may be due at least in part to an increasing sense of user competence with increasing levels of tool use.

From these insights, we note a number of limitations of the tools that could be addressed to improve the negative characteristics of user experience with underwater images. For example, as described in section "Pragmatic characteristics", auto-correction utilities for tone adjustments, white balancing and the removal of marine haze lead to a negative user experience as the outcomes can be inconsistent and/or unnatural and synthetic looking. This is consistent with section "Image enhancement software tools", where current research efforts into the automated enhancement of underwater images are found to be limited, as the metric(s) used by such techniques do not always align with human subjective judgement. Other limitations contributing to a characteristically negative user experience when enhancing underwater images as described by interview participants in section "Pragmatic characteristics" include difficulties achieving an imperceptible image spread with the graduated filter due to the characteristics of underwater images, and cloning underwater objects from marine backgrounds. From these insights and limitations we propose the following recommendations to improve the user experience for underwater photographers with image enhancement tools:

(a) An underwater image 'auto-judge' capability: Interview participant responses indicate they understand two broad aspects of a good underwater image, i.e. technical competence and aesthetic appeal. Technical competence refers to the extent to which degradations caused by the marine environment have been successfully corrected e.g. focus on the underwater subject is sharp, white balance is natural-looking, lighting and contrast are appropriate for an underwater environment, and marine occlusions (such as backscatter) are removed or at least do not distract from the image. Aesthetic appeal is more a matter of subjective underwater photographer opinion, although some underwater image patterns may be discernible e.g. where the subject of the image is a diver, swimming with eye contact towards the photographer and using artificial lighting to illuminate a point of interest in the foreground. Another underwater image pattern might be to capture the image as the diver exhales, bubbles of air ascending to the surface. An underwater image 'auto-judge' capability might address the negative user experience by providing comment on both image technical competence and aesthetic appeal. In this way, the underwater photographer is assisted with a (quasi) objective evaluation of the underwater image, which may enable the underwater photographer to decide if the image is worthy of further enhancement. By providing useful feedback 
about the image, the 'auto-judge' evaluation might also stimulate the underwater photographer, thus increasing enjoyment overall.

(b) An underwater image 'what-if modelling' capability: Interview participants noted that many underwater image enhancement packages record the history of changes to an image. However, it was difficult to 'clone-out' an area of interest (e.g. negative UtilityClone Stamp Tool) and not possible to keep various trials of composition in memory. Furthermore, autocorrection utilities for tone adjustments, white balancing and the removal of marine haze can lead to a negative user experience as outcomes can be unpredictable producing unnatural and synthetic looking underwater images. An underwater image 'what-if modelling' utility would stimulate the underwater photographer by keeping multiple developments of an image and their histories, produced by different preferred tool configurations, for side-by-side comparison.

\section{Limitations}

This paper has contributed novel insights into the user experiences of underwater photographers with image enhancement software. Whilst we endeavoured to make the study as rigorous as possible, the results may have benefited from conducting the questionnaire and interviews with larger sample sizes. This would have allowed us to cover a wider demographic of underwater photographers, in particular across a wider age range and balance of genders. In addition, there are some differences in the age distributions and operating systems used across questionnaire respondents and face-to-face interview participants. Nevertheless, both sample frames display a wide range of experience self-ratings and contain individuals with enough interest in the topic to become members of underwater imaging groups.

We acknowledge that for the face-to-face interviews, we chose to invite underwater photographers who enhance their images using Adobe products (Adobe Lightroom and Adobe Photoshop) which were found to be the most frequentlyused software in the questionnaire. This therefore limited the scope of the interviews to only exploring the characteristics of the user experience of these products. As the impact of input devices on user experience was not a focus of this study only limited data was collected regarding their general use. This and the small sample size of the study constrained us from drawing any firm conclusions on this subject and this topic is left open for future work.

Another potential limitation is that both the researchers and the interview participants are UK-based and as such this may have introduced a UK-centric bias. All questionnaires and face-to-face interviews were conducted in the
English language, which may also have introduced a bias in responses due to the language of participant communication. Replicating this study across other geographical regions (e.g. tropical waters) and languages may lead to different results, and so generalisation of the study findings to other sample populations may require reflection on the similarities and differences between the sample populations being studied.

\section{Conclusion}

In this study, we present our investigations into users' experience of image enhancement tools and packages for underwater images. We first conducted an online questionnaire to investigate what image enhancement software tools and packages are used by underwater photographers. Out of 81 world-wide respondents to the questionnaire, the majority response was for Adobe products i.e. 71 of the respondents $(87.7 \%)$ use either Adobe Lightroom or Adobe Photoshop. However, there was also a wide range of 19 other tools and packages used by individual respondents. Building on these results, and to investigate the characteristics of the image enhancement user experience for underwater photographers, seven questionnaire respondents with a variety of experience of Adobe products attended face-to-face interviews for detailed discussions relating to their use of image enhancement tools.

Qualitative coding of interview transcripts was centred on the frameworks of Hassenzahl [46, 49] with pragmatic and hedonic categories of codings for both positive and negative user experience. We conclude that one characteristic of users' experience with image enhancement tools for underwater images is a moderately negative experience overall. In addition, pleasure is an important characteristic of users' experience, indicating that a positive user experience is not necessarily confined to the functional pragmatics of completing the task. We also observe that the enjoyment of a positive experience appears to correlate positively with increasing levels of experience with the tools.

In an attempt to improve users' experience, we recommend that a number of software capabilities be developed for underwater photographers, including (1) an underwater image 'auto-judge' capability (incorporating an assessment of the technical competence of the underwater image along with its aesthetic appeal), and (2) an underwater image 'what-if' modelling capability. Future work will aim to characterise how underwater photographers subjectively assess their underwater imagery and then to develop underwater-specific image enhancement tools which are able to assess technical competence and aesthetic appeal. 
Acknowledgements We are grateful to the online questionnaire respondents and interview participants for their contributions to the study. We also thank Emma C. Ranger for her advice and helpful suggestions in the qualitative methodological aspects of this paper. This work was in part supported by the University of the West of England's Vice-Chancellor's Early Career Researcher Development Award.

\section{Declarations}

Conflict of interest On behalf of all authors, the corresponding author states that there is no conflict of interest.

Open Access This article is licensed under a Creative Commons Attribution 4.0 International License, which permits use, sharing, adaptation, distribution and reproduction in any medium or format, as long as you give appropriate credit to the original author(s) and the source, provide a link to the Creative Commons licence, and indicate if changes were made. The images or other third party material in this article are included in the article's Creative Commons licence, unless indicated otherwise in a credit line to the material. If material is not included in the article's Creative Commons licence and your intended use is not permitted by statutory regulation or exceeds the permitted use, you will need to obtain permission directly from the copyright holder. To view a copy of this licence, visit http://creativecommons.org/licenses/by/4.0/.

\section{References}

1. Ancuti C, Ancuti CO, Haber T, Bekaert P (2012) Enhancing underwater images and videos by fusion. In: 2012 IEEE conference on computer vision and pattern recognition (CVPR). IEEE, pp $81-88$

2. Anon (2020) Adobe Bridge. https://www.adobe.com/uk/produ cts/bridge.html. Accessed 03 Sep 2020

3. Anon (2020) Adobe Lightroom. https://www.adobe.com/uk/ products/photoshop-lightroom.html. Accessed 03 Sep 2020

4. Anon (2020) Adobe Photoshop. https://www.adobe.com/uk/ products/photoshop.html. Accessed 03 Sep 2020

5. Anon (2020) Affinity Photo. https://affinity.serif.com/en-gb/ photo/. Accessed 03 Sep 2020

6. Anon (2020) Apple Photos. https://www.apple.com/uk/macos/ photos/. Accessed 09 Sep 2020

7. Anon (2020) Aurora HDR. https://skylum.com/aurorahdr. Accessed 09 Sep 2020

8. Anon (2020) British Society of Underwater Photographers. http://www.bsoup.org. Accessed 09 Sep 2020

9. Anon (2020) Darktable. https://www.darktable.org/. Accessed 09 Sep 2020

10. Anon (2020) Digikam. https://www.digikam.org/. Accessed 09 Sep 2020

11. Anon (2020) Microsoft Photos. https://www.microsoft.com/ en-gb/p/microsoft-photos/9wzdncrfjbh4?activetab=pivot:overv iewtab. Accessed 09 Sep 2020

12. Anon (2020) Nik Collection. https://nikcollection.dxo.com/. Accessed 09 Sep 2020

13. Anon (2020) Nikon Capture NX-D. https://nikonimglib.com/ ncnxd/onlinehelp/en/index.html. Accessed 09 Sep 2020

14. Anon (2020) Noiseless. https://skylum.com/noiseless. Accessed 09 Sep 2020

15. Anon (2020) Nvivo. https://www.qsrinternational.com/nvivoqualitative-data-analysis-software/. Accessed 09 Sep 2020

16. Anon (2020) Photomatix. https://www.hdrsoft.com/ Accessed 09 Sep 2020
17. Anon (2020) PhotoLemur. https://photolemur.com/. Accessed 09 Sep 2020

18. Anon (2020) Picasa. https://picasa.google.co.uk/. Accessed 09 Sep 2020

19. Anon (2020) Pixeluvo. http://www.pixeluvo.com/. Accessed 09 Sep 2020

20. Anon (2020) PlayMemories. https://playmemoriesonline.com/? lang=en-gb. Accessed 09 Sep, 020

21. Anon (2020) RawTherapee. https://rawtherapee.com/. Accessed 09 Sep, 20

22. Anon (2020) Scottish Underwater Photographers Group. https:// www.facebook.com/groups/179191458941809/. Accessed 09 Sep, 2020

23. Anon (2020) SmartPhotoEditor. https://www.smartphotoeditor. com/. Accessed 09 Sep, 2020

24. Anon (2020) Snapseed. https://itunes.apple.com/gb/app/snaps eed/id439438619?mt=8. Accessed 09 Sep, 2020

25. Anon (2020) South Florida Underwater Photography Society. https://www.facebook.com/groups/sfupsclub/. Accessed 09 Sep, 2020

26. Anon (2020) Tonality. https://skylum.com/tonality. Accessed 09 Sep, 2020

27. Anon (2020) GNU Image Manipulation Program (GIMP). https://www.gimp.org/. Accessed 09 Sep, 2020

28. Anon (2020) Western Australia Underwater Photographic Society. https://www.facebook.com/groups/104593508800/. Accessed 09 Sep, 2020

29. Anon (2020) Wetpixel. http://wetpixel.com. Accessed 09 Sep, 2020

30. Anon (2021) Adobe Lightroom tutorials. https://helpx.adobe.com/ lightroom-cc/tutorials.html. Accessed 03 Mar, 2021

31. Anon (2021) Adobe Photoshop tutorials. https://helpx.adobe.com/ photoshop/tutorials.html. Accessed 03 Mar, 2021

32. Anon (2021) Adobe Photoshop Elements. https://www.adobe. com/uk/products/photoshop-elements.html. Accessed 04 Dec, 2021

33. Anon (2021) Qualtrics Online Survey Software. https://www.qualt rics.com/academic-solutions/uwe-bristol/. Accessed 04 Apr, 2021

34. Anon (2021) Silver Efex Pro. https://nikcollection.dxo.com/silverefex-pro/. Accessed 21 Sep, 2021

35. Anon (2021) Wacom. https://www.wacom.com/. Accessed 21 Sep, 2021

36. Anon (2021) Windows Photo Viewer. https://en.wikipedia.org/ wiki/Windows_Photo_Viewer. Accessed 13 Sep, 2021

37. Bailey R (2020) Bristol Underwater Photography Group. http:// www.bupg.co.uk. Accessed 09 Sep, 2020

38. Berman D, Treibitz T, Avidan S (2017) Diving into hazelines: color restoration of underwater images. In: Proceedingsof the British machine vision conference (BMVC), vol 1

39. Blair J, Czaja R, Blair E (2013) Designing surveys: a guide to decisions and procedures. Sage Publications, New York

40. Drews P, Nascimento E, Moraes F, Botelho S, Campos M (2013) Transmission estimation in underwater single images. In: Proceedings of the IEEE international conference on computer vision workshops, pp 825-830

41. Feather L (2020) Underwater photo editing for beginners. https://scubadiverlife.com/underwater-photo-editing-beginners/. Accessed 07 Sep 2020

42. Gatcum C (2016) The Beginner's Photography Guide. Penguin Random House, DK

43. Gibbs G (2007) Analysing qualitative data. Sage, London

44. Gietler S (2020) Underwater photography guide: 5 easy steps to process underwater photos. https://www.uwphotographyguide. com/underwater-photography-guide-beginners. Accessed 07 Sep. 2020 
45. Groves R, Fowler F Jr, Couper M, Lepkowski JM, Singer E, Tourangeau R (2011) Survey methodology, vol 561. Wiley, New York

46. Hassenzahl M (2003) The thing and I: understanding the relationship between user and product. In: Blythe M, Overbeeke K, Monk A (eds) Funology. Kluwer Academic Publishers, Dordrecht, pp $31-42$

47. Hassenzahl M (2008) User experience (UX): towards an experiential perspective on product quality. In: Proceedings of the 20th conference on l'Interaction Homme-Machine. ACM, pp 11-15

48. Hassenzahl M (2018) The thing and I (summer of' 17 remix). In: Funology 2. Springer, pp 17-31

49. Hassenzahl M (2018) The thing and I: understanding the relationship between user and product. In: Funology 2. Springer, pp 301-313

50. Hornbæk K, Hertzum M (2017) Technology acceptance and user experience: a review of the experiential component in HCI. ACM Trans Comput Hum Interact (TOCHI) 24(5):1-30

51. Islam MJ, Xia Y, Sattar J (2020) Fast underwater image enhancement for improved visual perception. IEEE Robot Autom Lett 5(2):3227-3234

52. ISO: ISO 9241-210:2019 ergonomics of human-system interaction-part 210: Human-centred design for interactive systems. https://www.iso.org/standard/77520.html (2019). Accessed 03 Sep, 2020

53. Jaffe JS (1990) Computer modeling and the design of optimal underwater imaging systems. IEEE J Ocean Eng 15(2):101-111

54. Karapanos E (2013) User experience over time. In: Modeling users' experiences with interactive systems. Springer, pp 57-83

55. Knijnenburg BP, Willemsen MC, Gantner Z, Soncu H, Newell C (2012) Explaining the user experience of recommender systems. User Model User Adap Int 22(4-5):441-504

56. Law ELC, Roto V, Hassenzahl M, Vermeeren AP, Kort J (2009) Understanding, scoping and defining user experience: a survey approach. In: Proceedings of the SIGCHI conference on human factors in computing systems, pp 719-728

57. Lazar J, Feng JH, Hochheiser H (2017) Research methods in human-computer interaction. Morgan Kaufmann, New York

58. Likert R (1932) A technique for the measurement of attitudes. Archiv Psychol

59. Liu R, Fan X, Zhu M, Hou M, Luo Z (2020) Real-world underwater enhancement: challenges, benchmarks, and solutions under natural light. IEEE Trans Circuits Syst Video Technol

60. Lythgoe J (1975) Problems of seeing colours under water. In: Vision in fishes. Springer, pp 619-634

61. Mobley CD (1994) Light and water: radiative transfer in natural waters. Academic Press, London

62. Moizer J, Lean J, Dell'Aquila E, Walsh P, Keary AA, O'Byrne D, Di Ferdinando A, Miglino O, Friedrich R, Asperges R et al (2019) An approach to evaluating the user experience of serious games. Comput Educ 136:141-151
63. Müller A, Anke S, Herrmann S, Katz P, Leuchtweis C, Miclau C, Wörner S, Korn O (2018) Measuring the influence of user experience on banking customers' trust. In: International conference on HCI in business, government, and organizations. Springer, pp 382-395

64. Panetta K, Gao C, Agaian S (2015) Human-visual-systeminspired underwater image quality measures. IEEE J Ocean Eng 41(3):541-551

65. Park J, Han SH, Kim HK, Cho Y, Park W (2013) Developing elements of user experience for mobile phones and services: survey, interview, and observation approaches. Human Factors Ergonom Manuf Serv Ind 23(4):279-293

66. Pizer SM (1990) Contrast-limited adaptive histogram equalization: speed and effectiveness stephen $\mathrm{m}$. pizer, r. eugene johnston, james p. ericksen, bonnie c. yankaskas, keith e. muller medical image display research group, vol 337. In: Proceedings of the first conference on visualization in biomedical computing, Atlanta, Georgia

67. Richards L (2014) Handling qualitative data: a practical guide. Sage, London

68. Ritter M, Winterbottom C (2017) UX for the Web: build websites for user experience and usability. Packt Publishing Ltd, New York

69. Saldaña $\mathbf{J}(2015)$ The coding manual for qualitative researchers. Sage, London

70. Santoso HB, Schrepp M (2019) The impact of culture and product on the subjective importance of user experience aspects. Heliyon 5(9): 02434

71. Seale C, Gobo G, Gubrium JF, Silverman D (2004) Qualitative research practice. Sage, New York

72. Sheldon KM, Elliot AJ, Kim Y, Kasser T (2001) What is satisfying about satisfying events? testing 10 candidate psychological needs. J Pers Soc Psychol 80(2):325

73. Tractinsky N, Katz AS, Ikar D (2000) What is beautiful is usable. Interact Comput 13(2):127-145

74. Uplavikar PM, Wu Z, Wang Z (2019) All-in-one underwater image enhancement using domain-adversarial learning. In: CVPR workshops, pp 1-8

75. Wigelius H, Väätäjä H (2009) Dimensions of context affecting user experience in mobile work. In: IFIP conference on humancomputer interaction. Springer, pp 604-617

76. Yang M, Sowmya A (2015) An underwater color image quality evaluation metric. IEEE Trans Image Process 24(12):6062-6071

Publisher's Note Springer Nature remains neutral with regard to jurisdictional claims in published maps and institutional affiliations. 